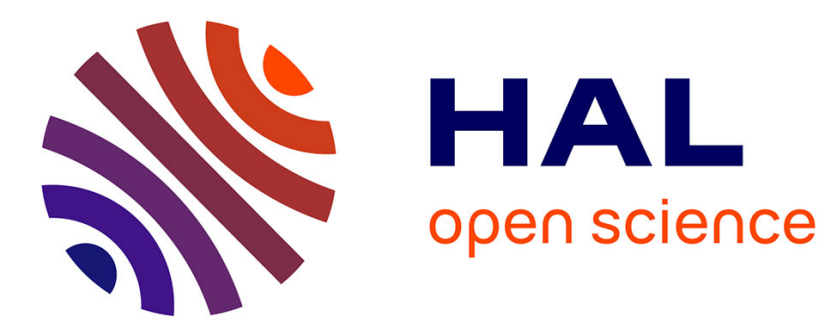

\title{
Solitary waves on superconfined falling liquid films
}

Gianluca Lavalle, Nicolas Grenier, Sophie Mergui, Georg F. Dietze

\section{To cite this version:}

Gianluca Lavalle, Nicolas Grenier, Sophie Mergui, Georg F. Dietze. Solitary waves on superconfined falling liquid films. Physical Review Fluids, 2020, 5, pp.032001. 10.1103/PhysRevFluids.5.032001. hal-02527784

\section{HAL Id: hal-02527784 \\ https://hal.science/hal-02527784}

Submitted on 7 Dec 2020

HAL is a multi-disciplinary open access archive for the deposit and dissemination of scientific research documents, whether they are published or not. The documents may come from teaching and research institutions in France or abroad, or from public or private research centers.
L'archive ouverte pluridisciplinaire HAL, est destinée au dépôt et à la diffusion de documents scientifiques de niveau recherche, publiés ou non, émanant des établissements d'enseignement et de recherche français ou étrangers, des laboratoires publics ou privés. 


\title{
Solitary waves on superconfined falling liquid films
}

\author{
Gianluca Lavalle* and Nicolas Grenier \\ Université Paris-Saclay, CNRS, LIMSI, 91400, Orsay, France. \\ Sophie Mergui and Georg F. Dietze ${ }^{\dagger}$ \\ Université Paris-Saclay, CNRS, FAST, 91405, Orsay, France.
}

(Dated: February 18, 2020)

\begin{abstract}
Solitary traveling waves are prominent features covering the surface of a falling liquid film and are known to promote heat/mass transfer. We focus on the little studied case where they are subject to an extremely confined counter-current gas flow, and we identify two novel secondary instabilities. At high gas velocities, a catastrophic instability develops, leading to flooding through wave reversal and liquid arrest. At lower gas velocities, an oscillatory instability occurs, producing a high-frequency periodic modulation of the wave height. Conjunction of this self-sustained oscillatory state and vortices forming in the liquid is shown to enhance mixing. We also show that the gas flow can cause extreme local film thinning, leading to almost dry patches where the liquid thickness is very small.
\end{abstract}

Flows through miniaturized channels are usually laminar and require active or passive methods to enhance mixing [1]. In the case of stratified two-phase flows, this can be achieved through flow structures resulting from interfacial instabilities [2]. In horizontal pressure-driven configurations, the instability is known to require a strong enough relative motion between the two phases [3,4]. By contrast, we study the vertical configuration of a gravity-driven falling liquid film in contact with an extremely confined gas, where the inertia-driven Kapitza instability [5] generates solitary surface waves that greatly enhance heat/mass transfer [6]. In this case, the gas velocity can be used as a free parameter to act on the waves.

Many works have studied the effect of a gas flow on the stability and dynamics of wavy falling liquid films [7-9]. Such studies have usually concentrated on weakly/moderately confined configurations. In that case, only the tangential interfacial viscous stresses exerted by the gas play a significant role, and turbulent conditions are required to affect meaningfully the liquid film [10]. By contrast, we focus on extreme confinement levels, where the gas flow and liquid film are of comparable thickness [11]. Here, gas pressure variations induced by surface waves become relevant [12], and the film is affected by the gas even under laminar conditions [13]. This configuration is bound to be more prone to flooding, i.e. occlusion of the channel by the liquid [14], wave reversal [8], or liquid arrest [10]. Yet, it is unclear how this unwanted phenomenon is provoked under extreme confinement. Does it result from a lack of saturated traveling-wave solutions or due to a loss of their stability? Answering such question may produce valuable insights for the design of falling film microreactors [15-17].

We have studied numerically solitary traveling waves under the effect of an extremely confined counter-current gas flow. For the conditions investigated, which represent real liquid properties and confinement levels typical of falling film microreactors, we find that flooding can be triggered at high gas velocities by a catastrophic secondary instability leading to wave reversal and liquid arrest. At lower gas velocities, we identify an oscillatory secondary instability, which causes a periodic (in time and space) modulation of the wave height. This self-sustained oscillatory state, in conjunction with vortical structures forming in the liquid, is found to enhance mixing within the solitary waves. It can be controlled through the gas velocity and thus represents an attractive approach to enhance inter-phase heat/mass transfer in small-scale devices. When the liquid volume and not the liquid flow rate is fixed, increasing the gas flow can cause pronounced film thinning, producing almost dry patches where the liquid thickness becomes extremely small.

We consider a two-dimensional liquid film falling down a vertical wall in interaction with a counter-current gas flow confined by a second wall at a distance $H^{\star}=1 \mathrm{~mm}$ (the star superscript denotes dimensional quantities). The working liquid (index l) corresponds to a $83 \%$ by weight aqueous dimethylsulfoxide (DMSO) solution typically used in experiments [18] and has density $\rho_{\mathrm{l}}=1098.3 \mathrm{~kg} / \mathrm{m}^{3}$, dynamic viscosity $\mu_{\mathrm{l}}=3.13 \mathrm{mPas}$, and surface tension $\sigma=0.0484$ $\mathrm{N} / \mathrm{m}$. The gas (index g) is air. We focus on solitary waves, i.e. composite structures consisting of a large tear-shaped hump and several capillary precursor ripples [19] (see graph 1b). Solitary waves are typically produced by nonlinear coarsening dynamics [20] that restructure the initial train of short waves emerging from linear wave selection, as the result of secondary subharmonic and/or sideband instabilities [21]. They produce maximal heat/mass transfer intensification in unconfined films [22].

We simulate this flow using the low-dimensional model of Dietze and Ruyer-Quil [12], which is based on the weighted residual integral boundary layer (WRIBL) approach [23, 24], accounts for the full coupling between the two phases,

\footnotetext{
* Author to whom correspondence should be addressed. gianluca.lavalle@limsi.fr; present address: Institut de Mécanique des Fluides de Toulouse (IMFT) - Univ. de Toulouse, CNRS-INPT-UPS, Toulouse, France.

† Author to whom correspondence should be addressed. dietze@fast.u-psud.fr
} 

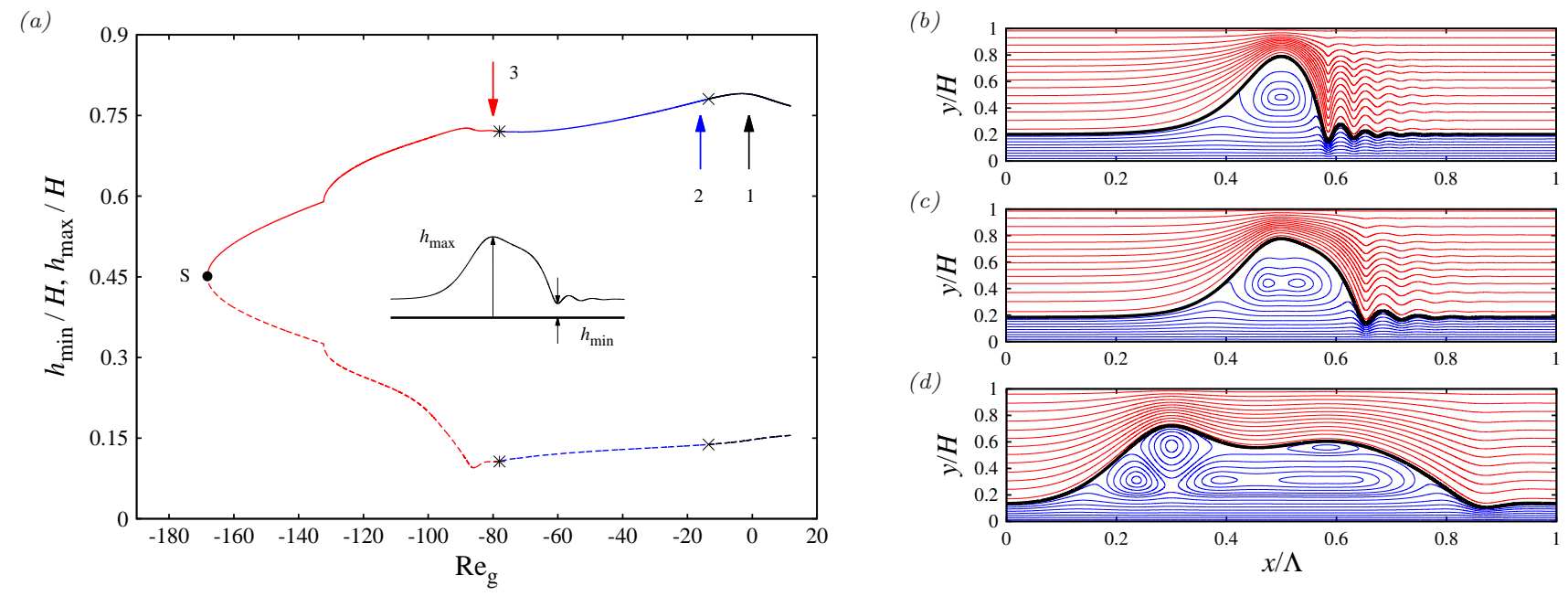

FIG. 1. Solitary traveling waves subject to an increasingly strong extremely confined gas flow: $H^{\star}=1 \mathrm{~mm} ; \mathrm{Ka}=509.5 ; \mathrm{Re}_{\mathrm{l}}=15$; $f=f_{0}=0.027$. (a) Effect of the gas Reynolds number $\operatorname{Re}_{\mathrm{g}}$ on the maximal height $h_{\max }$ (solid) and minimal height $h_{\text {min }}$ (dashed). Black (right of cross, $\operatorname{Re}_{\mathrm{g}}>-13.5$ ): stable solutions; blue (between cross and asterisk, $-78<\operatorname{Re}_{\mathrm{g}}<-13.5$ ): oscillatory instability; red (between asterisk and circle, $-168<\mathrm{Re}_{\mathrm{g}}<-78$ ): catastrophic instability. Limit point $\mathrm{S}$ coincides with linear stability cutoff. $(b-d)$ Streamlines in the reference frame of the wave for the three solutions marked by arrows in graph $a$. Thick lines represent film surface, blue streamlines represent the liquid (bottom), and red ones the gas (top): (b) $\operatorname{Re}_{\mathrm{g}}=-1 ;$ (c) $\operatorname{Re}_{\mathrm{g}}=-16$; (d) $\operatorname{Re}_{\mathrm{g}}=-80$.

and has been substantially validated [12]:

$$
\begin{aligned}
& \partial_{x} q_{1}+\partial_{t} h=0, \quad \partial_{x} q_{\mathrm{g}}-\partial_{t} h=0 \\
&\left\{S_{i} \partial_{t} q_{i}+F_{i j} q_{i} \partial_{x} q_{j}+G_{i j} q_{i} q_{j} \partial_{x} h\right\}=\left(1-\Pi_{\rho}\right)-\operatorname{Ka} \partial_{x x x} h+\left(C_{j 1}-\Pi_{\mu} C_{j \mathrm{~g}}\right) q_{j}+ \\
&\left\{J_{j} q_{j}\left(\partial_{x} h\right)^{2}+K_{j} \partial_{x} q_{j} \partial_{x} h+L_{j} q_{j} \partial_{x x} h+M_{j} \partial_{x x} q_{j}\right\},
\end{aligned}
$$

where the indices $i$ and $j$ should be permuted over the phase-indicators 1 and g using Einstein summation. All quantities have been rendered dimensionless using the scales $\mathcal{L}=\left(\mu_{1} / \rho_{1}\right)^{2 / 3} g^{-1 / 3}, \mathcal{U}=\left(\mu_{1} g / \rho_{1}\right)^{1 / 3}$, and $\mathcal{T}=\mathcal{L} / \mathcal{U}$. Further, $q_{1}$ and $q_{\mathrm{g}}$ designate the liquid and gas flow rates per unit width, $h$ is the film height, and the coefficients $S_{i}, F_{i j}, G_{i j}, C_{i j}$, $J_{j}, K_{j}, L_{j}$, and $M_{j}$ are known functions of $h$. The streamwise and crosswise coordinates are denoted $x$ and $y$, and $t$ denotes time. The dimensionless groups are the Kapitza number $\mathrm{Ka}=\sigma \rho^{1 / 3} g^{-1 / 3} \mu_{1}^{-4 / 3}$, the ratios of density and dynamic viscosity $\Pi_{\rho}=\rho_{\mathrm{g}} / \rho_{\mathrm{l}}$ and $\Pi_{\mu}=\mu_{\mathrm{g}} / \mu_{\mathrm{l}}$, and the Reynolds numbers $\operatorname{Re}_{\mathrm{l}}=q_{10}$ and $\operatorname{Re}_{\mathrm{g}}=q_{\mathrm{g} 0} \Pi_{\rho} / \Pi_{\mu}$, based on the nominal flow rates $q_{10}$ and $q_{\mathrm{g} 0}$ of the flat-film primary flow. For a counter-current gas flow, $q_{\mathrm{g} 0}$ and $\mathrm{Re}_{\mathrm{g}}$ are negative.

We solve numerically equation (1), either on a domain of wavelength $\Lambda$ subject to periodic boundary conditions, or on an open domain with inlet/outlet conditions, where waves of frequency $f$ are produced through inlet forcing. In both cases, the total flow rate $q_{\mathrm{tot}}=q_{\mathrm{l}}+q_{\mathrm{g}}$ is fixed. We also track traveling-wave solutions of (1) by numerical continuation [25] using the software Auto07P [26]. Such solutions are stationary in the reference frame $\xi=x$-ct moving with the wave speed $c$. We check their stability by using them as initial conditions for transient periodic computations. Stable solutions will remain unchanged in such computations, whereas unstable ones will evolve toward a different state. Details of the numerical procedures employed for solving the model equations (1) are given in [12].

To study the effect of the oscillatory instability on heat/mass transport (figure 3), we have also performed a direct numerical simulation (DNS) based on the full Navier-Stokes equations, which additionally solves a convectiondiffusion equation for the transport of a passive scalar. The solver employed for the DNS is Gerris [27] and details of the numerical setup are provided in [28].

Figure 1 illustrates how solitary traveling-wave solutions of fixed liquid Reynolds number $\operatorname{Re}_{1}$ and wave frequency $f=f_{0}=0.027$ evolve under the effect of an increasingly strong counter-current gas flow. This scenario is most practical from an experimental point of view [29]. Graph 1a displays the maximum $h_{\max }$ and minimum $h_{\min }$ wave height as a function of the gas Reynolds number Re $\mathrm{g}_{\mathrm{g}}$. The solid $\left(h_{\max }\right)$ and dashed $\left(h_{\min }\right)$ branches of the displayed curve join at the limit point $\mathrm{S}$, where the Kapitza instability is linearly suppressed and traveling-wave solutions disappear into the flat-film primary flow. The location of $\mathrm{S}, \mathrm{Re}_{\mathrm{g}}=-168$, can be predicted by linear stability analysis [13].

Graphs $1 \mathrm{~b}, 1 \mathrm{c}$, and $1 \mathrm{~d}$ represent streamlines in the reference frame of the wave for the solutions marked by arrows 1, 2, and 3 in graph 1a. As the gas velocity is increased, the surface wave is shaped into a flattened elongated liquid 

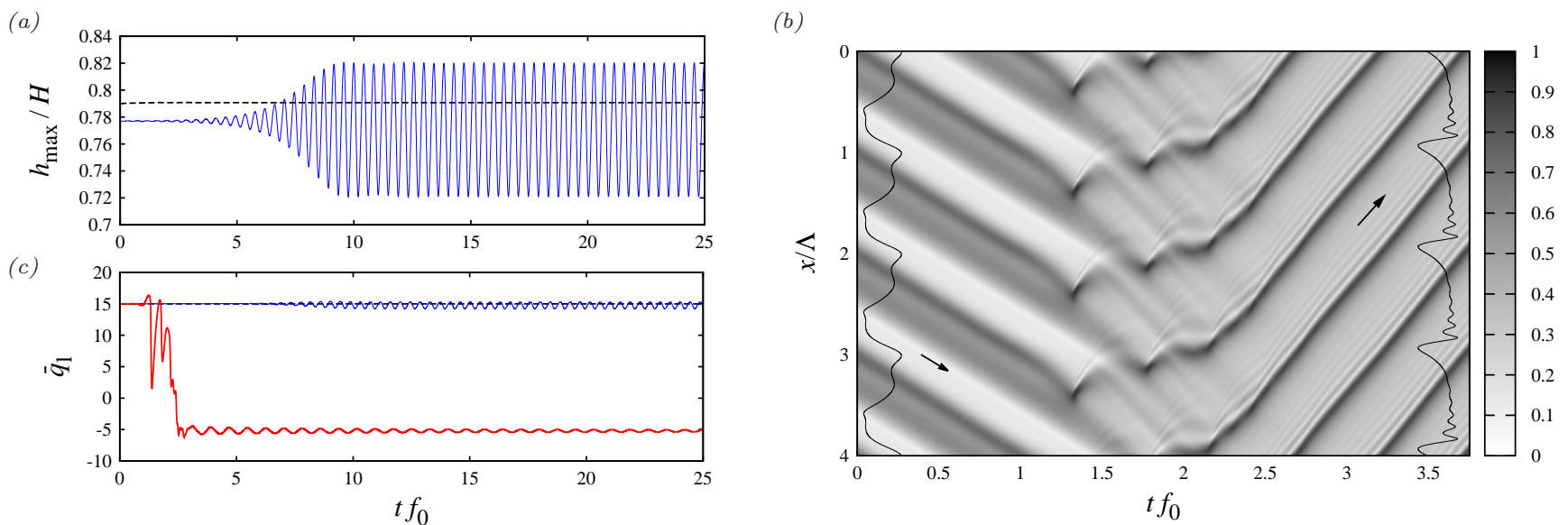

FIG. 2. Transient periodic computations started from traveling-wave solutions marked by arrows 1,2 , and 3 in graph 1 a. The total flow rate $q_{\text {tot }}$ and the liquid volume are fixed. (a) Time evolution of maximal wave height $h_{\text {max }}$ over several nominal wave periods $1 / f_{0}$. Black dashed: stable regime (arrow 1); blue solid: oscillatory instability regime (arrow 2); (b) space-time plot of film height contours $h(x, t) / H$ for the regime of catastrophic instability (arrow 3), displaying wave reversal. Profiles superimposed on left and right correspond to start and end times; (c) time-traces of the wave-averaged liquid flow rate $\bar{q}_{1}=\Lambda^{-1} \int_{0}^{\Lambda} q_{1} d x$ for computations from graphs $a$ (black and blue lines) and $b$ (thick red line), showing liquid arrest for the catastrophic instability.

hump containing an increasing number of counter-rotating vortices. These result from the subdivision of the single vortex in graph $1 b$, which is typically observed in roll waves [30].

We now check the stability of traveling-wave solutions by advancing them numerically in time through transient periodic computations, as represented in figure 2 . In these computations, $q_{\text {tot }}$ and the liquid volume (due to periodicity) are fixed, and thus the wave-averaged liquid flow rate $\bar{q}_{1}=\Lambda^{-1} \int_{0}^{\Lambda} q_{l} \mathrm{~d} x$ may vary in time w.r.t. the traveling-wave solution used as initial condition. We find that traveling-wave solutions on the black curve segment in graph 1a $\left(\operatorname{Re}_{\mathrm{g}}>-13.5\right)$ are stable. This is shown in graph 2a, representing $h_{\max }$ time traces from different transient computations. The black dashed line corresponds to a computation started from the traveling-wave solution at $\operatorname{Re}_{\mathrm{g}}=-1$ (arrow 1 in graph 1a), and we see that $h_{\max }$ does not change over time. On the blue curve segment in graph $1 \mathrm{a},-78<\operatorname{Re}_{\mathrm{g}}<-13.5$, traveling-wave solutions are subject to an oscillatory secondary instability. This produces a high-frequency selfsustained modulation of the wave height, as evidenced by our transient computation in graph 2a (blue solid line), which was started from a traveling-wave solution at $\mathrm{Re}_{\mathrm{g}}=-16$ (arrow 2 in graph 1a). We see that $h_{\max }$ oscillates in time with a secondary frequency $f_{\mathrm{s}} \approx 3 f_{0}$ much greater than the nominal frequency $f_{0}$. The amplitude of the oscillation first grows exponentially, proving that oscillations result from linear instability, and then saturates to a constant value.

On the red curve segment in graph $1 \mathrm{a},-168<\mathrm{Re}_{\mathrm{g}}<-78$, traveling-wave solutions are subject to a secondary instability that leads to a catastrophic event. This is shown in graph $2 \mathrm{~b}$, representing a space-time diagram of the film thickness $h(x, t) / H$, for a transient computation started from the traveling-wave solution at $\operatorname{Re}_{\mathrm{g}}=-80$ (arrow 3 in graph 1a). The profile superimposed on the left represents the initial condition $h(x, t=0)$. In the early stages, $t f_{0}<1$, this profile propagates downstream without changing significantly. Then, for $1 \leq t f_{0} \leq 2.5$, a reversal of the wave occurs under the effect of the counter-current gas flow. Finally, for $t f_{0}>2.5$, a new upward traveling wave is formed (profile superimposed on the right). Importantly, the observed wave reversal coincides with liquid arrest, as evidenced by the corresponding time trace of $\bar{q}_{1}$, represented by a thick red line in graph $2 \mathrm{c}$, which shows the wave-averaged liquid flow rate to become negative. In a real system, where the liquid inlet flow rate is fixed, wave reversal and liquid arrest are usually associated with flooding (supplementary movie [31] shows how these phenomena unfold in a spatially evolving computation). Thus, based on graph 1a and figure 2, we may conclude that flooding in the studied configuration is triggered by a secondary instability, whereas traveling-wave solutions never cease to exist, up to the linear instability cutoff (point $\mathrm{S}$ in graph 1a).

We now concentrate on the oscillatory instability observed for $-78<\operatorname{Re}_{\mathrm{g}}<-13.5$. Figure 3 shows how this instability develops spatially, based on transient computations on an open domain with inlet/outlet conditions. Here, $\mathrm{Re}_{\mathrm{l}}$ is imposed through the time-averaged liquid inlet flow rate $\tilde{q}_{1}(x=0)=q_{l 0}$, which is subjected to a small harmonic temporal perturbation of frequency $f_{0}$. This allows forcing the desired solitary waves, as is typically done in experiments [29].

Graphs 3a and 3b represent computations at $R_{g}=-19$ and $R_{g}=-25$. The black lines represent snapshots of the film profile (Eulerian view), whereas the red lines trace the path followed by the wave crests as time progresses (Lagrangian 

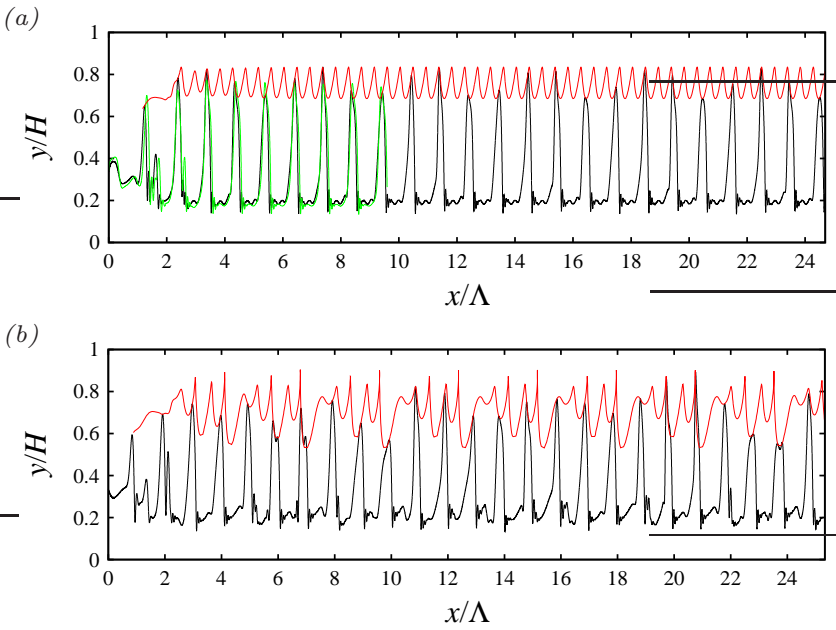

(c)

(d)
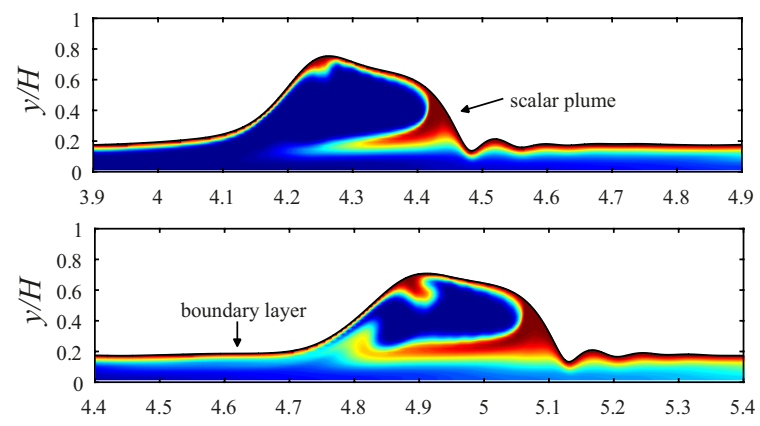

(e)

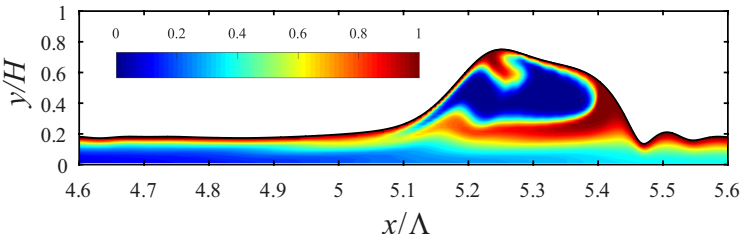

FIG. 3. Regime of oscillatory instability. $(a, b)$ Transient computations on an open domain with inlet/outlet conditions: $H^{\star}=1$ $\mathrm{mm}, \mathrm{Re}_{\mathrm{l}}=15, f_{0}=0.027$. Displayed length is $0.8 \mathrm{~m}$. Black lines: snapshots of the film profile $y=h(x, t=\mathrm{const})$ (Eulerian view); red: path followed by the wave maxima, as the oscillating wave humps propagate through the domain (Lagrangian view); green: snapshot from DNS. (a) $\mathrm{Re}_{\mathrm{g}}=-19$ : single-mode oscillation; (b) Re $\mathrm{g}_{\mathrm{g}}=-25$ : multi-mode oscillation. (c-e) Heat/mass transport data obtained from DNS in graph $a$ : $\mathrm{Pe}=4590$. See also supplementary movie [32]. Instantaneous color plots of the field of the transported scalar $\Theta$ over the course of one nominal wave period $1 / f_{0}$. (c) $t f_{0}=0 ;(d) t f_{0}=0.65 ;(e) t f_{0}=1.0$.

view). The oscillatory nature of these paths implies that the wave height of a given solitary wave traveling through the domain undergoes a self-sustained periodic oscillation (in time and space). In graph 3a, the oscillation consists of a single mode with a wavelength about twice smaller than the nominal separation of the solitary waves. In graph $3 \mathrm{~b}$, where the gas flow is stronger, the oscillation is enriched by additional modes making it more choppy, but it remains periodic. In both cases, the amplitude of the oscillation is substantial. The green profile in graph 3a corresponds to our DNS, performed on a shorter domain, and supplementary movie [32] shows that this DNS also displays oscillations.

The present oscillatory instability differs from the secondary sub-harmonic and side-band instabilities observed for liquid films falling in a quiescent atmosphere [21]. Those instabilities apply to trains of short periodic waves and result from wave interaction, leading to wave coalescence. The amplitude modulations they induce are of longer and not shorter length than the nominal wavelength. Importantly, these instabilities do not require any interaction between the gas and the wavy liquid film, whereas our instability is precisely caused by such an interaction.

The conjunction of the oscillatory instability (figure 3) and the vortices within the solitary waves (graph 1b) enhances convective heat/mass transport in the liquid film. To show this, we have solved a convection-diffusion equation for the representative scalar $\Theta$ in our DNS of the oscillatory regime in graph 3a. We have set $\Theta=1$ at the gas/liquid interface, neglecting the transport resistance in the gas, and $\Theta=0$ at the liquid inlet. The Péclet number, relating convective to diffusional transport, is $\mathrm{Pe}=\mathcal{U L} / \alpha=4590$, where $\alpha$ denotes the diffusivity of $\Theta$ in the liquid.

The color plots in graphs 3c, 3d, and 3e represent the instantaneous $\Theta$ field at successive time points over the course of one nominal wave period $1 / f_{0}$. We focus on the thin scalar plume that is seen to grow from the leading toward the trailing part of the wave in the successive snapshots. It results from the stagnation point flow generated by vortices within the wave (see e.g. graph 1b). This flow destroys the interfacial $\Theta$ boundary layer at the front of the wave but compresses the one at the wave back. The latter mechanism is responsible for the significant enhancement of inter-phase heat/mass transfer due to solitary waves [22], but usually breaks down once the growing plume has reached the rear part of the wave [28]. In our case, the oscillatory instability prevents this from happening, by causing a repeated folding of the plume as it approaches the wave back (graphs 3d and 3e). We had already observed in graphs $1 \mathrm{~b}$ to $1 \mathrm{~d}$ the capacity of the gas to rearrange vortical structures within the wave. The supplementary movie [32] shows the plume folding dynamics in action. As a result of this mechanism, the interfacial boundary layer growing into the upstream residual film remains thin, and this increases the overall heat/mass transfer to the liquid.

We shall discuss now how solitary traveling waves behave under an increasing gas flow, when not $\mathrm{Re}_{\mathrm{I}}$ but the liquid volume is fixed. Such a situation may arise within the pulmonary capillaries [33], where films of secreted mucus are subject to the respiratory air flow. We find that traveling waves can break down due to an extreme local thinning of the liquid film. Figure 4 shows how this occurs for a solitary wave of fixed wavelength $\Lambda=46.8 H$ and liquid volume (per unit width) $V_{1}=0.31 \Lambda H$. Graph 4 a maps film thickness profiles $h(x)$ as a function of the gas Reynolds number $\mathrm{Re}_{\mathrm{g}}$ (vertical axis), using grey scales. Graph $4 \mathrm{~b}$ represents the corresponding evolution of the minimal film thickness $h_{\text {min }}$. As the gas velocity is increased, the thin regions of the liquid film are increasingly drained, eventually forming 
(a)

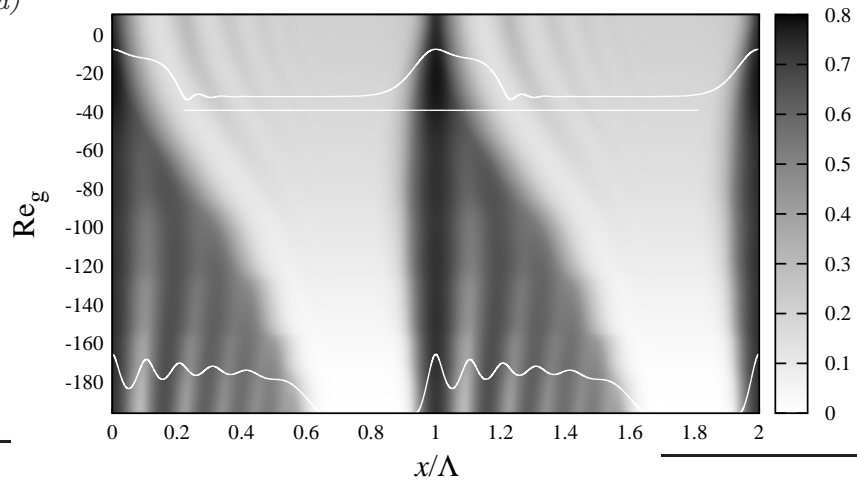

(b)

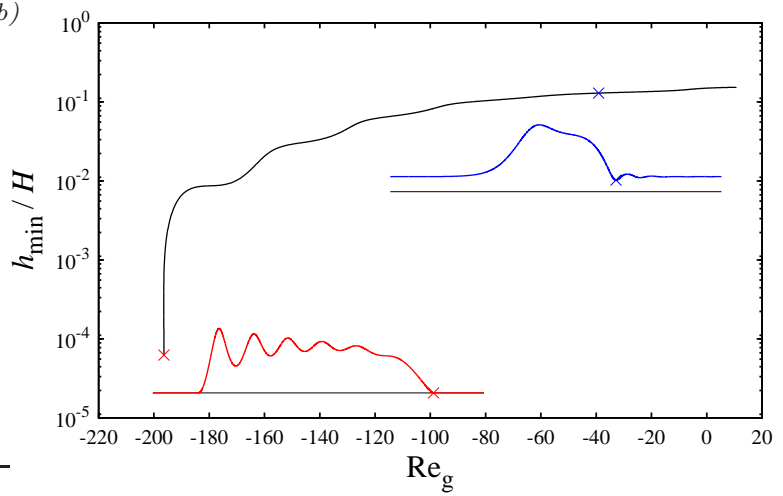

FIG. 4. Extreme film thinning under an increasing counter-current gas flow. Traveling-wave solutions at fixed $\Lambda=46.8 H$ and liquid volume (per unit width) $V_{\mathrm{l}}=0.31 \Lambda H$. (a) Grey scale plot of film surface profiles $h(x) / H$ as a function of Reg (vertical axis); (b) corresponding evolution of the minimal film thickness $h_{\text {min }}$ (vertical axis is logarithmic). The lowest thickness reached is $h_{\min }^{\star}=62 \mathrm{~nm}$. In both graphs, two representative cross-sectional views are also included.

patches that are almost dry. Our numerical continuation breaks down at $\operatorname{Re}_{\mathrm{g}}=-196.3$, having reached $h_{\mathrm{min}}^{\star}=62 \mathrm{~nm}$, which is not far beyond the range of long-range van der Waals forces [34]. It is interesting that purely hydrodynamic phenomena lead the film to this critical state, which may be conceived as negative flooding, i.e. the gas "touching" the wet wall instead of the liquid "touching" the dry wall. Of course, to check for actual film rupture, intermolecular interaction properties such as disjoining pressure and wall wettability need to be accounted for [35]. Integrating such a description [36] into our model, might be an interesting problem for future work.

In this letter, we have explored a new region of parameter space by considering superconfined wavy falling liquid films in contact with a counter-current gas flow. We have focused on a scenario in which the liquid flow rate and frequency of surface waves are fixed while the gas flow velocity is increased, which is most plausible from an experimental perspective. And, our computations were performed for an existing liquid [18]. We find that traveling-wave solutions exist no matter how strong the gas flow is, up until the point where the primary instability disappears. However, depending on the gas velocity, they are subject to two different secondary instabilities. At high velocities, the instability is catastrophic and produces flooding through wave reversal and liquid arrest. At lower velocities, the instability is oscillatory, producing a high-frequency periodic (in time and space) modulation of the wave height. This self-sustained oscillation is found to intensify convective transport within the liquid film and thus represents an attractive control mechanism to enhance inter-phase heat/mass transfer. We have also found a scenario in which the effect of a counter-current gas flow generates extremely thin patches within the liquid film.

We conclude by evaluating the feasibility of experiments. Zhang et al. [15] have produced experimentally stratified water/air flows in falling-film microreactors, where the liquid flowed in $0.3 \mathrm{~mm}$ deep parallel grooves of width $W^{\star}=1$ $\mathrm{mm}$, with its surface pinned on either side to the top of the grooves [37], and the gas confined by an upper wall at $H^{\star}=0.8 \mathrm{~mm}$. In our case, pinning should be avoided to allow for the motion of the liquid-gas interface on the side walls. Then, the contact angle $\phi$ comes into play, and this limits the channel width $W$ in regard to forming a liquid annulus or rupturing the film laterally. Also, $W$ should be sufficiently small to limit formation of 3-dimensional surface waves $[38,39]$. At the same time, it should be as large as possible, in order to limit the stabilizing effect of the side-wall meniscus on the interfacial instability [40]. For water films on PMMA (Polymethylmethacrylate), steel, or PDMS (Polydimethylsiloxane), channel widths of $W=5-10 \mathrm{~mm}$ should allow producing the desired conditions. For the DMSO-water solution used in our computations, the contact angle on PDMS is $\phi=93^{\circ}$ [41], and this reduces the ability of the liquid to climb the side walls and the stabilizing effect of the meniscus [40], relaxing several constraints.

\section{ACKNOWLEDGMENTS}

We appreciate helpful discussions with J. P. Hulin. The work was supported by the ANR wavyFILM project, grant ANR-15-CE06-0016-01 of the French Agence Nationale de la Recherche.

[1] H. A. Stone, A. D. Strook, and A. Ajdari, Engineering flows in small devices: Microfluidics toward a lab-on-a-chip, Annu. Rev. Fluid Mech. 36 (2004). 
[2] X. Hu and T. Cubaud, Viscous wave breaking and ligament formation in microfluidic systems, Physical Review Letters 121 (2018).

[3] C.-S. Yih, Instability due to viscosity stratification, Journal of Fluid Mechanics 27, 337 (1967).

[4] S. G. Yiantsios and B. G. Higgins, Linear stability of plane poiseuille flow of two superposed fluids, Physics of Fluids 31, 3225 (1988).

[5] P. L. Kapitza, Wave flow of thin layer of viscous fluid (in Russian), Zhurn. Eksper. Teor. Fiz. 18, 3 (1948).

[6] P. N. Yoshimura, P. Nosoko, and T. Nagata, Enhancement of mass transfer into a falling laminar liquid film by twodimensional surface waves-some experimental observations and modeling, Chemical Engineering Science 51, 1231 (1996).

[7] S. V. Alekseenko, S. P. Aktershev, A. V. Cherdantsev, S. M. Kharlamov, and D. M. Markovich, Primary instabilities of liquid film flow sheared by turbulent gas stream, International Journal of Multiphase Flow 35, 617 (2009).

[8] D. Tseluiko and S. Kalliadasis, Nonlinear waves in counter-current gas-liquid film flow, Journal of Fluid Mechanics 673, 19 (2011).

[9] Y. Y. Trifonov, Instabilities of a gas-liquid flow between two inclined plates analyzed using the navier-stokes equations, International Journal of Multiphase Flow 95, 144 (2017).

[10] Y. Y. Trifonov, Counter-current gas-liquid wavy film flow between the vertical plates analyzed using the Navier-Stokes equations, AIChE Journal 56, 1975 (2010).

[11] S. A. Setu, R. P. A. Dullens, A. Hernández-Machado, I. Pagonabarraga, D. G. A. L. Aarts, and R. Ledesma-Aguilar, Superconfinement tailors fluid flow at microscales, Nat. Commun. 6:7297 (2014).

[12] G. F. Dietze and C. Ruyer-Quil, Wavy liquid films in interaction with a confined laminar gas flow, J. Fluid Mech. 722 (2013).

[13] G. Lavalle, Y. Li, S. Mergui, N. Grenier, and G. F. Dietze, Suppression of the kapitza instability in confined falling liquid films, Journal of Fluid Mechanics 860, 608 (2019).

[14] N. A. Vlachos, S. V. Paras, A. A. Mouza, and A. J. Karabelas, Visual observations of flooding in narrow rectangular channels, International Journal of Multiphase Flow 27, 1415 (2001).

[15] H. Zhang, G. Chen, J. Yue, and Q. Yuan, Hydrodynamics and mass transfer of gas-liquid flow in a falling film microreactor, AIChE Journal (2009).

[16] F. Seebauer, P. Poechlauer, S. Braune, and S. Steinhofer, Tube bundle falling film microreactor for performing gas liquid reactions, US Patent 8221708B2 (2012).

[17] A. Lapkin and P. T. Anastas, eds., Green Chemical Engineering, Handbook of Green Chemistry, Vol. 12 (Wiley-VHC, 2018).

[18] G. F. Dietze, F. Al-Sibai, and R. Kneer, Experimental study of flow separation in laminar falling liquid films, Journal of Fluid Mechanics 637, 73 (2009).

[19] S. V. Alekseenko, V. E. Nakoryakov, and B. G. Pokusaev, Wave Flow of Liquid Films (Begell House, 1994).

[20] H. C. Chang, E. A. Demekhin, E. Kalaidin, and Y. Ye, Coarsening dynamics of falling-film solitary waves, Physical Review E 54, 1467 (1996).

[21] J. Liu and J. P. Gollub, Onset of spatially chaotic waves on flowing films, Physical Review Letters 70, 2289 (1993).

[22] A. Rastaturin, E. Demekhin, and E. Kalaidin, Optimal regimes of heat-mass transfer in a falling film, Journal of NonEquilibrium Thermodynamics 31, 1 (2006).

[23] C. Ruyer-Quil and P. Manneville, Improved modeling of flows down inclined planes, European Physical Journal B 15, 357 (2000).

[24] S. Kalliadasis, C. Ruyer-Quil, B. Scheid, and M. G. Velarde, Falling Liquid Films, edited by S. S. Antman, P. Holmes, L. Sirovich, and K. Sreenivasan, Applied Mathematical Sciences, Vol. 176 (Springer, 2012).

[25] E. L. Allgower and K. Georg, Introduction to Numerical Continuation Methods, Classics in Applied Mathematics (SIAM, 2003).

[26] E. J. Doedel, AUTO07P: Continuation and bifurcation software for ordinary differential equations, Montreal Concordia University (2008).

[27] S. Popinet, An accurate adaptive solver for surface-tension-driven interfacial flows, J. Comput. Phys. 228, 5838 (2009).

[28] G. F. Dietze, Effect of wall corrugations on scalar transfer to a wavy falling liquid film, Journal of Fluid Mechanics 859, 1098 (2019).

[29] N. Kofman, S. Mergui, and C. Ruyer-Quil, Characteristics of solitary waves on a falling liquid film sheared by a turbulent counter-current gas flow, Int. J. Multiph. Flow 95 (2017).

[30] S. V. Alekseenko, V. A. Antipin, A. V. Bobylev, and D. M. Markovich, Application of PIV to velocity measurements in a liquid film flowing down an inclined cylinder, Experiments in Fluids 43, 197 (2007).

[31] See Supplemental Material at [URL will be inserted by publisher] for a movie of a computation with our WRIBL model. Parameters correspond to those in graph 3a, only that the gas flow rate was increased over time until producing wave reversal and liquid arrest, upon which the computation broke down. These phenomena are triggered around Reg $=-65$, somewhat below the threshold $R_{\mathrm{g}}=-78$ for the catastrophic instability of traveling-wave solutions (see graph 1a), owing to wave coalescence events.

[32] See Supplemental Material at [URL will be inserted by publisher] for a movie of the direct numerical simulation in graphs 3a (green curve), 3c, 3d, and 3e.

[33] J. Grotberg, Respiratory fluid mechanics, Physics of Fluids 23, 021301 (2011).

[34] J. Israelachvili, Intermolecular and Surface Forces (Academic Press, 2011).

[35] D. Bonn, Wetting and spreading, Review of Modern Physics 81 (2009).

[36] U. Thiele, M. G. Velarde, K. Neuffer, and Y. Pomeau, Sliding drops in the diffuse interface model coupled to hydrodynamics, 
Physical Review E 64, 061601 (2001).

[37] M. Al-Rawashdeh, V. Hessel, P. Löb, K. Mevissen, and F. Schönfeld, Pseudo 3-d simulation of a falling film microreactor based on realistic channel and film profiles, Chemical Engineering Science 63, 5149 (2008).

[38] N. Kofman, S. Mergui, and C. Ruyer-Qui, Three-dimensional instabilities of quasi-solitary waves in a falling liquid film, Journal of Fluid Mechanics 757, 854 (2014).

[39] G. F. Dietze, W. Rohlfs, K. Nährich, R. Kneer, and B. Scheid, Three-dimensional flow structures in laminar falling liquid films, Journal of Fluid Mechanics 743, 75 (2014).

[40] T. Pollak, A. Haas, and N. Aksel, Side wall effects on the instability of thin gravity-driven films - from long-wave to short-wave instability, Physics of Fluids 23, 094110 (2011).

[41] Z. Zhang, W. Wang, A. N. Korpacz, C. R. Dufour, Z. J. Weiland, C. R. Lambert, and M. T. Timko, Binary liquid mixture contact-angle measurements for preciseestimation of surface free energy, Langmuir 35, 12317 (2019). 